\title{
New rapid identification test for Clostridium difficile
}

\author{
S T Aspinall, S F Dealler
}

\begin{abstract}
Aims: A set of five tests were developed and tested for their ability to confirm the identity of $C$ difficile colonies within $\mathbf{3 0}$ minutes.

Methods: The relevant substrates were incorporated into four filter paper squares attached to a plastic carrier (Diffstrip), five enzymes/products (prolyl aminopeptidase, galactosidase, leucine aminopeptidase, acid phosphatase and indole). The strips were inoculated, incubated for 20 minutes, and reagents added.

Results: $96.4 \%$ (212 of 220 ) strains of $C$ difficile were immediately differentiated from 51 other Clostridium spp tested. The remaining $3.6 \%$ (eight of 220 ) of $C$ difficile isolates produced a reaction pattern similar to some of the Clostridium sporogenes tested and required additional tests. None of the other Clostridium spp tested produced reaction patterns similar to $C$ difficile.

Conclusion: The Difistrip allowed colonies of $C$ difficile to be confirmed within 30 minutes for $96.4 \%$ of isolates, with less than $4 \%$ requiring any additional tests. No strains of $\boldsymbol{C}$ difficile were misidentified and no strains of other Clostridium spp tested were misidentified as $\boldsymbol{C}$ difficile.
\end{abstract}

(F Clin Pathol 1992;45:956-958)

The pathogenic role of Clostridium difficile in the aetiology of intestinal disease has been well documented, ${ }^{1-3}$ causing almost all cases of pseudomembranous colitis (PMC) and about $25 \%$ of cases of antibiotic associated diarrhoea (AAD). ${ }^{45}$ Laboratory diagnosis of such diarrhoea predominantly consists of culture of the organism from faeces or detection of either toxin $A$ by enzyme immunoassay ${ }^{6}$ or toxin $B$ by tissue culture. ${ }^{7}$ Although toxin detection correlates best with the clinical infection, ${ }^{8}$ culture is still regarded as a highly sensitive method ${ }^{9}$ and is required for epidemiological purposes.

Presumptive identification can easily be made by both smell (horse-dung smell) and Chartreuse-green fluorescence under long wave $(360 \mathrm{~nm})$ ultraviolet light. ${ }^{10}$ Confirmation is then often made by one of three techniques. First, gas liquid chromatography (GLC) has been widely reported as the standard method of identification, ${ }^{112}$ but is relatively time consuming and requires both expensive equipment and expertise. A second method requires commercially obtained bio- chemical identification strips, such as Minitek anaerobe II (Becton Dickinson, Oxford) or API 20A (API bioMèrieux, Basingstoke, ${ }^{13} 14$ but these may take up to 72 hours to perform and may not be reproducible. A third and more recent method is the use of enzyme based identification strips, such as RapID ANA II (Mercia Diagnostics Ltd, Guildford), ATB 32A (API bioMèrieux) API ZYM (API bioMèrieux), or An-Ident (API bioMèrieux) 15-17 $^{17}$ which offer reproducible results, but as $C$ difficile is relatively unreactive in such systems, supplementary tests may be required and misidentification can occur. This paper describes the development of a rapid (less than 30 minutes), accurate, and highly reproducible method for confirmation of $C$ difficile isolates.

\section{Methods}

TEST STRIPS

All test strips were supplied by Lab $M$. They consisted of a plastic carrier $(65 \mathrm{~mm} \times 12$ $\mathrm{mm}$ ) with attached squares of filter paper which were impregnated with dried test substrates. Numerous identification tests were investigated for their potential use (unpublished data), with five tests finally being chosen. These were incorporated on to the same strip (Diffstrip) which carried four filter paper squares (two tests are combined into one square). They detected the following enzymes (substrates indicated in parentheses):

(1) Acid phosphatase (a naphthol phosphate ( Lab M) and sodium nitrite buffered to $\mathrm{pH} \mathrm{5.0}$ using phosphate/citrate buffer).

(2) Indole (p-dimethylaminocinnamaldehyde (DMCA) (Sigma Chemical Co Ltd, Poole) and toluene sulphonic acid (Sigma Ltd)).

(3) Leucine aminopeptidase (leucine- $\beta$-naphthylamide) (Lab M).

(4) $\beta$-galactosidase and prolyl aminopeptidase (5 bromo, 4-chloro, 3-indolyl $\beta$-galactosidase and prolyl- $\beta$-napthylamide) (Bachem Ltd, Saffron Walden).

ORGANISMS TESTED

Strains $(n=220)$ of $C$ difficile (including NCTC 11204 and NCTC 11209), eight strains of $C$ sporogenes (including NCTC 6929 , NCTC 8594, NCTC 276 and NCTC 532), nine strains of $C$ sordellii (including NCTC 8780), seven strains of $C$ bifermentans (including NCTC 6801, NCTC 6927, and NCTC 
506), five strains of $C$ septicum, eight strains of $C$ perfringens, seven strains of $C$ glycolicum, one strain of $C$ histolyticum (ATCC 4259), one strain of $C$ tetani, one strain of $C$ cadaveris, one strain of $C$ tertium, one strain of $C$ beijerinkii and one strain of $C$ butyricum. NCTC strains were derived from the National Collection of Type Cultures, Central Public Health Laboratory, Colindale, and ATCC 4259 was supplied by the American Type Culture Collection, Centers for Disease Control, Atlanta, USA.

\section{TEST PROTOCOL}

The test organisms were derived from national collections or from the culture collection at Preston Public Health Laboratory. They were identified using standard methods ${ }^{18}$ and grown on Columbia agar (Unipath Ltd, Basingstoke) with $7 \% \mathrm{v} / \mathrm{v}$ whole horse blood, cycloserine cefoxitin fructose agar (CCFA) (Unipath Ltd), and moxalactam/norfloxacin selective agar (CDMN), ${ }^{19}$ for either 24 or 48 hours at $37^{\circ} \mathrm{C}$ in an anaerobic cabinet. After incubation the acid phosphatase, leucine aminopeptidase, and $\beta$-galactosidase/prolyl aminopeptidase filter paper test squares were inoculated with a colony of the test organism using a bacteriological loop (sufficient to observe a visible inoculum on the strip). The indole test was supplied with a lighter but still visible inoculum. Each test square was then moistened with a loopful of sterile distilled water and the result of the indole test recorded after 30 seconds (a positive result taken as a blue-purple colour around the edge of the inoculum). The strip was then incubated aerobically at $37^{\circ} \mathrm{C}$ for 20 minutes in a humidified chamber after which time the result of the $\beta$-galactosidase test was recorded (presence of $\beta$-galactosidase results in the formation of an azure blue colour) before adding a drop of reagent $A(10 \mathrm{mg} / \mathrm{ml}$ of sulphanilic acid (Aldrich Chemical Co Ltd, Gillingham) in $5 \mathrm{~N}$ acetic acid) to the acid phosphatase test, and a drop of reagent $B$ (1 $\mathrm{mg} / \mathrm{ml}$ of DMCA in $\mathrm{IN}$ hydrochloric acid) to the $\beta$-galactosidase/prolyl aminopeptidase test and the leucine aminopeptidase test. The

Diffstrip results for 271 strains of Clostridium sPP

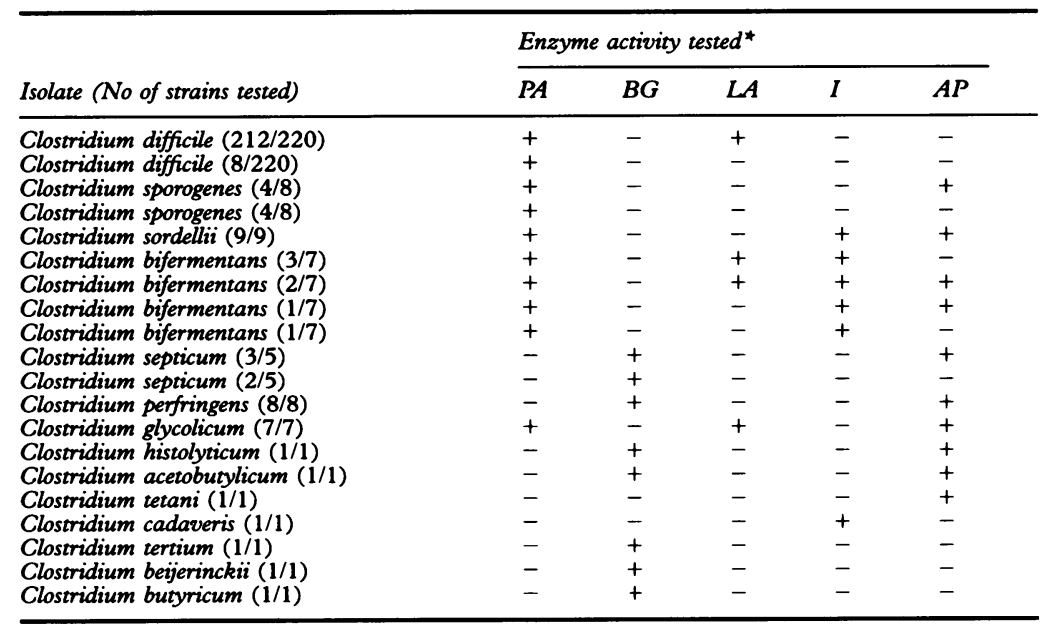

^PA - Prolyl aminopeptidase; BG - $\beta$-Galactosidase; LA - Leucine aminopeptidase; I - Indole production; AP - Acid phosphatase. results of these were then recorded after five minutes of incubation at room temperature. Phosphatase activity was detected by the formation of an orange colour while the presence of prolyl aminopeptidase activity was indicated by a red-pink colour and that of leucine aminopeptidase by a pink colour at the site of the inocula.

\section{Results}

The table shows the results obtained from the five Diffstrip tests (acid phosphatase, indole, leucine aminopeptidase, $\beta$-galactosidase and prolyl aminopeptidase) for the 271 strains of Clostridium spp investigated. All strains of $C$ difficile produced identical test results with the exception of eight isolates $(3.6 \%)$ which yielded negative leucine aminopeptidase reactions. All other isolates of $C$ difficile produced positive prolyl aminopeptidase and leucine aminopeptidase tests, with negative $\beta$-galactosidase, indole, and acid phosphatase results.

All strains of $C$ sordellii could easily be differentiated from $C$ difficile by their positive acid phosphatase and indole reactions. All strains of $C$ bifermentans tested produced indole, together with three of seven of the isolates also yielding a positive acid phosphatase reaction. Fifty per cent of the strains of $C$ sporogenes could be differentiated from $C$ difficile by their positive acid phosphatase reactions. The remaining $50 \%$ (four of eight) resulted in only a positive prolyl aminopeptidase reaction, which is similar to the $3.6 \%$ of leucine aminopeptidase negative $C$ difficile strains and would therefore require a supplementary test. All other strains of Clostridium spp tested could also be differentiated from $C$ difficile from the configuration of the positive reactions (table).

There was no difference in results obtained whether cultures were grown on blood agar, CCFA, or CDMN, after either 24 or 48 hours of anaerobic incubation.

\section{Discussion}

The new generation of anaerobic identification systems are now predominantly chromogenic enzyme detection tests. These offer results after four hours of incubation, are highly reproducible, and require no additional facilities, such as gas liquid chromatographs. Unfortunately, due to the unreactive nature of many Clostridium spp in terms of the substrates available within these commercial systems, identification may only be to genus level and misidentification can occur.

Investigation of patients with antibiotic associated diarrhoea often entails faecal culture and identification of suspect colonies as $C$ difficile. The identification of Clostridium spp other than $C$ difficile under these circumstances is unnecessary; therefore, a system which rapidly and accurately confirms $C$ difficile from other clostridia would save time spent in fully identifying isolates. From the results of investigating numerous rapid tests for differentiation of various Clostridium spp (unpublished 
data) five adequately identified $C$ difficile from other clostridia. Five tests were incorporated on to the Diffstrip, which required a maximum of 20 minutes' incubation and therefore fulfills these criteria. The prolyl aminopeptidase test, although not helping to differentiate the $C$ sporogenes/C sordellii/C bifermentans/C glycolicum group from $C$ difficile, not only acted as a positive control test for those clostridia which remained unreactive with all other tests, but aided separation of $C$ difficile from the other Clostridium spp tested.

The $\beta$-galactosidase test was similar in that all the aforementioned group of clostridia did not produce $\beta$-galactosidase, but the test allowed most other Clostridium sp tested to be differentiated rapidly (no reagents need to be added).

The indole test permitted differentiation of $C$ sordellii and $C$ bifermentans from $C$ difficile, and the incorporation of the leucine aminopeptidase test into the strip allowed $96.4 \%$ (212 of 220) of $C$ difficile isolates tested to be differentiated from all other Clostridium spp tested.

The acid phosphatase test separated $C$ sordellii and $C$ glycolicum from $C$ difficile, together with $50 \%$ of $C$ sporogenes isolates tested. The remaining $50 \%$ of $C$ sporogenes produced a similar reaction pattern to $3.6 \%$ of the $C$ difficile strains, therefore, on the rare occasions that such a reaction profile occurs, an additional test may be required for final differentiation, such as lipase production. $C$ sporogenes should rarely cause problems as its growth should be inhibited if faeces are cultured on selective media. ${ }^{20}$

As very little growth of suspect colonies is required to perform the test (unlike the commercially available enzyme based systems), identification can be carried out directly from isolated colonies on the primary culture plate, thereby further reducing the time needed for confirmation. The Diffstrips allowed rapid confirmation of $96.4 \%$ of isolates of $C$ difficile and clearly indicated those that required additional testing.
We thank Dr D N Hutchinson for his support for the work and his comments on the manuscript.

1 George RH, Symonds JM, Dimock F, et al. Identification of Clostridium difficile as a cause of pseudomembranous Clostridium difficile as a cause
colitis. Br Med $\mathcal{J} 1978 ; 1: 695$.

2 Lyerly DM, Krivan HC, Wilkins TD. Clostridium difficile: its disease and toxins. Clin Microbiol Rev 1988;1:1-18.

3 Borriello SP, Davies HA, Kamiya S, Reed PJ, Seddon, S. Virulence factors of Clostridium difficile. Rev Infect Dis 1990;12 (Suppl 2):S185-S91.

4 Gilligan PH, McCarthy LR, Genta VM. Relative frequency of Clostridium difficile in patients with diarrhoeal disease. $f$ Clin Microbiol 1981;14:26-31.

5 Burdon DW. Spectrum of disease, In: Borriello SP, ed. Antibiotic associated diarrhoea and colitis. Boston: Martinus Nijhoff Publishers, 1984:9-25.

6 Di Persio JR, Varga FJ, Conwell DL, Kraft JA, Kozak KJ, Willis DH. Development of a rapid enzyme immunoassay for Clostridium difficile toxin $A$ and its use in the diagnosis of $\mathrm{Cl}$. difficile-associated disease. $f$ Clin Microbiol 1991;29:2724-30.

7 Burdon DW. Laboratory investigation of antibiotic-associated diarrhoea. Association of Clinical Pathologists Broadsheet 102. London: ACP/BMA, 1982.

8 Walker RC, Ruane PJ, Rosenblatt JE, et al. Comparison of culture, cytotoxicity assays and enzyme-linked immunosorbent assay for toxin $A$ and toxin $B$ in the diagnosis of Clostridium difficile related enteric disease. Diagnost Microbiol Infect Dis 1986;5:61-9.

9 Gerding DN, Olson MM, Peterson LR, et al. Prospective case-controlled epidemiologic study of $\mathrm{Cl}$ difficile-associated diarrhoea and colitis in adults. Arch Intern Med 1986;146:95-100.

10 George WL, Sutter VL, Citron D, Finegold SM. Selective and differential medium for isolation of Clostridium and differential medium for isolation

11 Berg JD, Mills RG, Coleman DJ. Improved gas liquid chromatography method for identification of Clostridium difficile. I Clin Pathol 1985;38:108-13.

12 Levett PN, Phillips KD. Gas chromatography identification of Clostridium difficile and detection of cytotoxin from a modified selective medium. I Clin Pathol 1985;38:82-5.

13 Gresser ME, Shanholtzer CJ, Gerding DN, Garret CR, Peterson LR. Evaluation of the 24h API 20A anaerobe system for identification of Clostridium difficile. $\mathcal{J}$ Clin Microbiol 1984;19:915-6.

14 Bate G. Comparison of Minitek anaerobe II, API An-Ident, and RapID ANA systems for identification of Clostridium difficile. Am $\mathcal{f}$ Clin Pathol 1986;85:716-9.

15 Levett PN. Identification of Clostridium difficile using the API ZYM system. Eur $₹$ Clin Microbiol 1985;4:505-7.

16 Head CB, Ratnam S. Comparison of API ZYM system with API AN-Ident, API 20A, Minitek anaerobe II, and RapID-ANA systems for identification of Clostridium difficile. $\mathcal{F}$ Clin Microbiol 1988;26:144-6.

17 Quentin C, Desailly-Chanson MA, Bebear C. Evaluation of AN-Ident. f Clin Microbiol 1991;29:231-5.

8 Allen SD, Baron EJ. Clostridium. In: Barlows A, Hausler WJ, Herrmann KL, Isenberg HD, Shadomy HJ, eds. Manual of Clinical Microbiology. Washington, DC: American Society for Microbiology, 1991:505-21.

19 Aspinall ST, Hutchinson DN. A new selective medium for the isolation of Clostridium difficile from faeces. $\mathcal{f}$ Clin Pathol 1992;45:812-5.

20 Borriello SP, Honour P. Detection, isolation and identification of Clostridium difficile. In: Borriello SP, ed. Antibiotic associated diarrhoea and colitis. Boston: Martinus biotic associated diarrhoc
Nijhoff, 1984:38-47. 